Forum

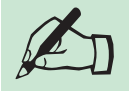

\section{Tarifvergleich TARMED 1.1 zu SLK}

In seinem Tarifvergleich stellt Herr Baumgartner [1] den TARMED in einem rosaroten Licht dar, indem er einerseits vorwiegend die wenigen im Vergleich zum SLK ähnlich dotierten invasiven Positionen aufführt und andererseits einen SLK-TPW angibt, der nicht den Realitäten entspricht. Korrekt für den Vergleich wäre ein TPW SLK von Fr. 4.95 und eine infrastrukturelle Abgabe für Belegärzte/-ärztinnen (BÄ) von 30\%. Abgabeanteile von $70 \%$ beziehen sich vornehmlich auf Privathonorare von Chefärz- ten/-ärztinnen, die im übrigen fixbesoldet für Leistungen im Grundversicherungsbereich keine Rechnung stellen können. Des weiteren sei an dieser Stelle festgestellt, dass auch im SLK verschiedene v.Xa. zeitlich kurzdauernde invasive Leistungen für BÄ nicht kostendeckend sind. Ohne Anspruch auf (auch arztnahe) TL-Anteile, die für ambulante invasive Leistungen im TARMED vollumfänglich dem Spital zufliessen, muss der BA seine Praxisinfrastruktur, die leerstehend in der Regel mehr als Fr. 200.- pro Stunde beträgt, aus seinem Honorar bestreiten, wobei nochmals zu betonen ist, dass im TARMED die AL immer inkl. Assistenz ist, d.h. dass der BA aus der AL auch seinen resp. seine Assistenten zu entschädigen hat. Dies veranlasste FMH-Präsidenten, Dr. med. H. H. Brunner, vor noch nicht zu langer Zeit jeweils zur Bemerkung, die BÄ seien im TARMED eben systemfremd. An dieser Tatsache hat sich seit den GRAT-Arbeiten leider nichts geändert. Im übrigen ist der Tarifvergleich von Herrn Baumgartner auch deshalb problematisch, da wohl kaum von einem TARMED-TPW von Fr. 1.ausgegangen werden kann. Im folgenden wird anhand des Kapitels 21 aufgezeigt, dass viele und v.a. häufig durchgeführte invasive Leistungen künftig nicht mehr von BÄ erbracht werden können, da kaum noch Stundenumsätze von Fr. 200.- erreicht werden könnten. Im Gegensatz zu Herrn Baumgartner werde ich dabei die Betreuung des hospitalisierten Patienten bei stationären Eingriffen sogar korrekt anführen (alle Tarifvergleiche gemäss Vorgaben Tarifdienst FMH).

Plastische Zirkumzision (TM zu SLK -39,73\%) / ambulant $\leq 24$ Stunden

\begin{tabular}{llclll}
\hline TARMED & & \multicolumn{3}{c}{ SLK $4.95-30 \%$} & Neis \\
\hline Nummer & Text & Preis AL & Nummer & Text & Preis \\
\hline 00.0210 & & 44.12 & 2476.00 & Plast. Phimosenoperation & 259.87 \\
\hline 21.2680 & Zirkumzision jede Methode & 112.51 & & & \\
\hline Total & & 156.63 & Total & & 259.87 \\
\hline
\end{tabular}

TUR - Prostata (TM zu SLK - 61,75\%) / Hospitalisationsdauer 5 Tage

\begin{tabular}{|c|c|c|c|c|c|}
\hline \multicolumn{3}{|l|}{ TARMED } & \multicolumn{3}{|c|}{ SLK $4.95-30 \%$} \\
\hline Nummer & Text & Preis $A L$ & Nummer & Text & Preis \\
\hline $3 \times 00.0210$ & $\begin{array}{l}\text { Betreuung des hospitalisierten Patienten } \\
\text { Kategorie A }\end{array}$ & $3 \times 44.12=132.36$ & 2470.00 & Prostataresektion & 1126.12 \\
\hline $2 \times 00.0230$ & $\begin{array}{l}\text { Betreuung des hospitalisierten Patienten } \\
\text { Kategorie B }\end{array}$ & $2 \times 22.06=44.12$ & & & \\
\hline 21.2180 & Ablatio prostatae jede Methode & 254.20 & & & \\
\hline Total & & 430.68 & Total & & 1126.12 \\
\hline
\end{tabular}

Kryptorchismusoperation einseitig (TM zu SLK -26,3\%) / ambulant $\leq 24$ Stunden

\begin{tabular}{|c|c|c|c|c|c|}
\hline \multicolumn{3}{|l|}{ TARMED } & \multicolumn{3}{|c|}{ SLK $4.95-30 \%$} \\
\hline Nummer & Text & Preis $A L$ & Nummer & Text & Preis \\
\hline 00.0210 & $\begin{array}{l}\text { Betreuung des hospitalisierten Patienten } \\
\text { Kategorie A }\end{array}$ & 44.12 & 2477.00 & $\begin{array}{l}\text { Kryptorchismusoperation } \\
\text { einseitig }\end{array}$ & 433.12 \\
\hline 21.3130 & $\begin{array}{l}\text { Operative Versorgung bei Kryptorchismus, } \\
\text { einseitig }\end{array}$ & 275.03 & & & \\
\hline Total & & 319.15 & Total & & 433.12 \\
\hline
\end{tabular}

Herr Baumgartner deckt in seinem Tarifvergleich zudem vielleicht sogar unbewusst eine Interpretationsproblematik auf. Aufgrund der Interpretationen (TARMED 1.1 GI 40) sind die Zuschläge für die hospitalisierten Patienten sinngemäss klar nur für diese und nicht für die ambulanten Patienten vorgesehen, d.h. dass die Positionen "Betreuung des hospitalisierten Patienten" (00.210 und 00.0230, wie auch 00.0310) bei ambulanten Patienten (Hospitalisation definitionsgemäss weniger als 24 Stunden) nicht verrechnet werden dürften, analoge Posi- tionen wurden für ambulante Patienten bezeichnenderweise nicht geschaffen, und man muss davon ausgehen, dass die Versicherer aufgrund bisher gemachter Erfahrungen kaum daran interessiert wären, dies nachzuholen!

In der jetzt vorliegenden TARMED-Version wäre die Entschädigung des Belegarztes für seine operative Tätigkeit im ambulanten Bereich somit noch geringer als oben aufgeführt. Diese Konstellation zeigt nochmals deutlich auf, dass die Existenz v.a. der BAe bei Annahme der aktuellen TARMED-Ver- sion aufs höchste und in jeder Hinsicht gefährdet wäre. Es geht daher nicht an, dass derartige Missstände erst im Rahmen der Tarifpflege irgendwann nach Einführung des TARMED angegangen werden. Derartige Unkorrektheiten müssen zwingend jetzt bzw. vor der definitiven Annahme des TARMED beseitigt werden.

\section{A. Eijsten, W. Gartenmann}

1 Baumgartner M. Tarifvergleich TARMED $1.1 \mathrm{zu}$ SLK. Schweiz Ärztezeitung 2002;83(7):306-8. 


\section{Replik}

Die Verfasser des Leserbriefes weisen im Zusammenhang mit der Hospitalisation auf einen wichtigen Punkt hin, nämlich den Geltungsbereich von TARMED. Grundsätzlich ist TARMED nur für den ambulanten Bereich Tarifgrundlage. Im Unfallversicherungsbereich kennt die SUVA aber Abmachungen mit Belegarztspitälern, nach denen die ärztliche Leistung mittels Einzelleistungstarif ("Blauer Tarif») und die Hospitalisation mit einer Tagespauschale (Hotellerie) abgerechnet wird. In diesen Fällen könnte - eine Änderung der Vertragsverhältnisse nicht vorausgesetzt - auch bei stationären Patienten TARMED zur Anwendung kommen. Was die Festsetzung des zu vergleichenden Taxpunktwertes betrifft, so ist bei der in der Schweiz geltenden Vielfalt von Taxpunktwerten und Infrastrukturabgaben kein allein seligmachender Vergleich zu bewerkstelligen. Jeder Belegarzt muss für sich, in Kenntnis seiner Verhältnisse und auch in Kenntnis der Abrechnungsmechanismen von TARMED die entsprechenden Tarifvergleiche berechnen. In jedem Fall sei aber davon abgeraten, nur einzelne Leistungen zu vergleichen. Bei einem Vergleich müssen alle Leistungen sowohl nach aktuellem Tarif, als auch nach TARMED berücksichtigt werden.

Markus Baumgartner, Oberkirch

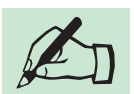

Weshalb ich gegen TARMED gestimmt habe Allen Kamelhengsten wurde mitgeteilt, dass ihre alten eisernen Nasenringe, auch in ihrem eigenen Interesse, ersetzt werden müssen. In gemeinsamen Verhandlungen habe man sich auf ein vernickeltes Modell geeinigt, das vielleicht aus Gründen der Kostenneutralität etwas enger sein werde. Sie sollten die Massnahme befürworten, sonst würde sie einfach verfügt werden, was einen wichtigen Verlust an Freiheit bedeutete. Zudem würden sie nach einer Ablehnung kaum mehr als Verhandlungspartner ernstgenommen. Einige fanden dies eine Farce und stimmten deshalb nein.

Dr. med. Peter Baumann, Zürich

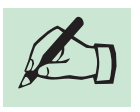

TARMED: et maintenant?

La vie n'est pas une fatalité - TARMED non plus! Ainsi donc, 54\% des membres de la FMH se sont prononcés sur ce nouveau système tarifaire, une majorité confortable des votants acceptant tant la nomenclature que les conventions y relatives. Ce résultat me parait regrettable, mais il est là et l'on s'y fera. $46 \%$ d'abstentions pour un sujet touchant pourtant de près notre activité quotidienne (et la compliquera; quel beau progrès!), cela reflète un important malaise qui ne s'explique pas que par la peur de la nouveauté ou de "l'inconnu».

Comme à l'occasion de toute campagne, les arguments raccourcis, parfois fallacieux ou simplistes n'ont pas manqué, particulièrement ces dernières semaines. Dont celui qui prétendait que TARMED viendrait de toute façon - à défaut d'être négocié, il serait imposé tel quel.

Tous les médecins ou presque s'accordent pourtant à reconnaître que l'édifice pose d'importants problèmes, y compris le président le la FMH, le Dr H. H. Brunner. Alors? Il ne nous resterait que le choix entre suicide et exécution?

C'est une vision fataliste de notre rôle dans la société, la vision de qui pense avoir perdu, quoi qu'il arrive. Certes, la société a beaucoup changé ces dernières années et avec elle la valeur du médecin. Or, la véritable question n'est pas qui perd ou qui gagne, mais dans quel système de santé nous nous reconnaissons. Celui qui vend des pièces des "prestations" selon la terminologie officielle - ou celui qui accorde une attention (médicale, psychologique et humaine) au patient. Qui a choisi ce dernier engagement, ne s'attardera pas davantage sur TARMED, mais défendra les intérêts des patients contre le mercantilisme. La conscience professionnelle devrait - me semble-t-il - nous inciter à accorder la priorité aux droits humains - dont le droit à la santé - plutôt qu'à la liberté de commerce.

Nous avons perdu plus de 10 ans et beaucoup d'énergie à vouloir déplacer une mer de glace, mais il n'est pas trop tard pour changer de cap. Cessons de ne nous occuper que du fonds de commerce et abordons plutôt les vrais problèmes de santé. Ce combat pour une médecine humaniste sera seul à même de restreindre les velléités de limiter le droit des patients par tarif médical interposé.

Dr M. Herrmann, Onex

\section{Pressemitteilung}

Auf den Spuren von Pollen, Milben, Nüssen $\mathrm{Ct}$ Co.

Vom 25. Februar bis 4. Mai 2002 ist das Schweizerische Zentrum für Allergie, Haut und Asthma (aha!) mit einem Team von Fachärzten und Krankenschwestern auf Allergietour durch die Deutschschweiz. Der mobile Allergieteststand von aha! macht Halt in zehn grossen Einkaufszentren. Je eine Woche lang. An Ort können Interessierte kostenlos ihr persönliches Allergierisiko abklären lassen, Beratung und Information zum Thema Allergien einholen.

Nach 15 Minuten wissen die Testpersonen Bescheid: ob eine Neigung zu allergischen Reaktionen ersichtlich ist; oder ob der Befund bereits auf eine bestimmte Allergie hinweist und weitere Abklärungen beim Facharzt oder in einer Allergologieklinik angezeigt sind. Bei rund 60 Prozent der Testpersonen sind erfahrungsgemäss keine verdächtigen Spuren auszumachen. Sie erhalten eine Diagnose der erfreulichen Art. aha! hat die Allergietour in Partnerschaft mit der Schweizerischen Gesellschaft für Allergologie und Immunologie (SGAI) organisiert. Vorgaben und Abläufe des Allergierisikotests wurden gemeinsam erarbeitet. Vertreter der ärztlichen Fachorganisation stehen während der Tour beratend im Einsatz. Informationshungrige finden am aha!Stand ausserdem eine ganze Reihe von Broschüren zu den verschiedenen allergischen Krankheitsbildern.

aha! Schweizerisches Zentrum für Allergie, Haut und Asthma, www.ahaswiss.ch 


\section{Pressemitteilungen}

\section{Eröffnung der "Spacebar» auf} www.alles-im-griff.ch

Junge Menschen sind heute gesundheitlich besonders gefährdet, weil sie immer früher Hochprozentiges konsumieren. Dass ein vernünftiger Umgang mit Alkohol jedoch keine trockene Angelegenheit sein muss, beweist das Bundesamt für Gesundheit auf der neu gestalteten Website www.alles-imgriff.ch. Neben vielen Informationen, Links und Anregungen lockt auch das neue Gratis-Download-Spiel «Spacebar».

"Alles im Griff?» startet ins vierte Kampaund einem packenden Präventionsspiel zum Herunterladen. Die Besucherinnen und Besucher finden auf der Homepage ein vielfältiges Angebot im Zusammenhang mit Alkohol: Hilfe, Ideen und Anregungen, Informationen und weiterführende Links, eine Übersicht über die Präventionskampagnen und vor allem auch Fun. Das Präventionsprogramm zum verantwortungsbewussten Umgang mit Alkohol wird vom Bundesamt für Gesundheit (BAG), der Eidgenössischen gnenjahr mit einem neuen Internet-Auftritt
Alkoholverwaltung (EAV) sowie der Schweizerischen Fachstelle für Alkohol- und andere Drogenprobleme (SFA) getragen.

\section{Treffpunkt "Spacebar»}

Kühlen Kopf bewahren heisst es, wenn sich die Nachtschwärmer und Fernfahrer in der turbulenten "Spacebar" zu einem Stelldichein treffen. Der Spieler, die Spielerin, die sich von den Barkeepern überlisten lassen und ihren Alkoholkonsum nicht kontrollieren, erfahren hautnah die fatalen Auswirkungen von Alkohol wie verminderte Zielsicherheit und langsameres Reaktionsvermögen. Die braucht es aber, um Punkte zu sammeln und ein Highscore zu erzielen, der im weltweiten Wettbewerb mithalten kann. Das Gratis-Download-Spiel "Spacebar» stammt aus der Küche von Phenomedia (Catch the sperm). Die Schweizer Milchproduzenten SMP haben das Spiel mitfinanziert. Durch die Zusammenarbeit mit SMP können wertvolle Synergien genutzt und die Jugendlichen noch besser angesprochen werden.

Download über www.alles-im-griff.ch

\section{Bundesamt für Gesundheit}

\section{Schweizer Knochenmarkspenden} sind begehrt

Seit 1988 haben sich im Schweizer Register für Knochenmarkspender über 18000 freiwillige Spender/innen eintragen lassen. Gemessen an der Bevölkerungszahl belegt die Schweiz im internationalen Vergleich damit einen mittleren Platz. Nun ist im Jahresbericht der World Marrow Donor Association nachzulesen, dass unser Land bei der Gegenüberstellung von Anzahl Knochenmarkstammzellspenden $\mathrm{zu}$ registrierten Spendern/-innen weltweit vordere Ränge einnimmt. Aus der Erhebung, die insgesamt 38 Länder erfasst, darf man schliessen, dass Schweizer Knochenmarkstammzellspender/innen begehrt sind. Dies lässt sich damit begründen, dass sich Schweizer Spender/innen durch besondere Merkmale auszeichnen:

- rund 99 Prozent aller im Schweizer Register eingetragenen Spender/innen sind bereits HLA-DR-typisiert, was das Suchen nach passenden Spenden zuverlässiger macht und die Zeiten somit wesentlich verkürzt;

- Schweizer Spender/innen verfügen zum Teil über HLA-Gewebekombinationen, wie sie auf der Weit nur selten oder gar nur einmal vorkommen;

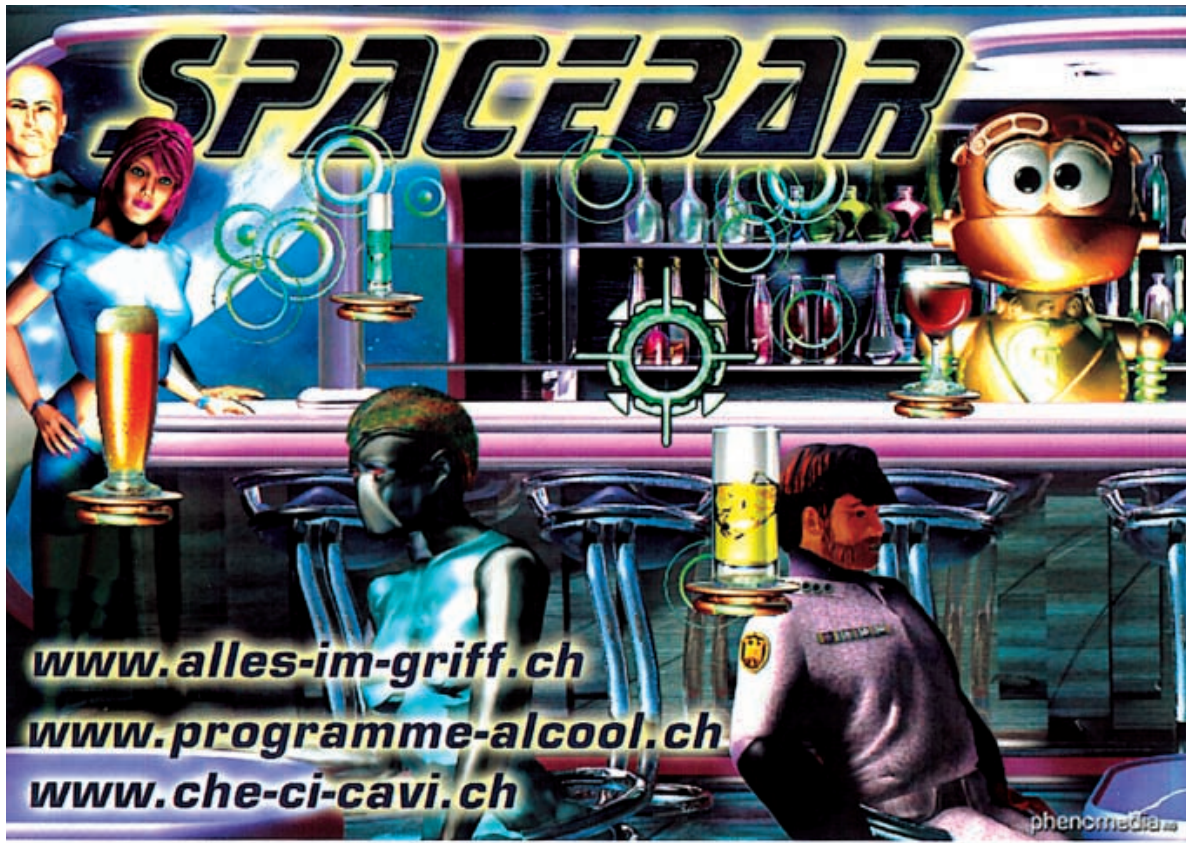

und "last but not least" zeichnen sich Schweizer Spender/innen durch ihre grosse Verfügbarkeit und Spendebereitschaft aus.

Dass sich das Schweizer Register über das Ergebnis des internationalen Vergleichs freut, ist verständlich. Denn es ist eine Bestätigung für das jahrelange Bestreben zur Erreichung und Erhaltung eines hohen Qualitätsstandards. Dank und Anerkennung gebühren jedoch den über 18000 freiwilligen Spendern/-innen in unserem Land. Wer zum Thema Knochenmarkstammzellen mehr Informationen sucht, findet diese auf der Homepage www.knochenmarkregister.ch.

Margrit Kern, Geschäftsführerin

Stiftung Schweizer Register für

Knochenmarkspender, Tel. 03196076 36,

E-Mail: info@swissregistry.ch 